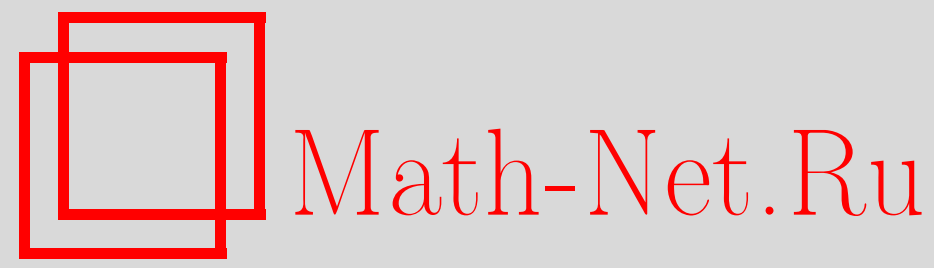

А. М. Шойтов, Структурно эквивалентные цепочки в равновероятной полиномиальной схеме, Матем. вопр. криптогр., 2012, том 3, выпуск 3, 129-151

DOI: https://doi.org/10.4213/mvk64

Использование Общероссийского математического портала Math-Net.Ru подразумевает, что вы прочитали и согласны с пользовательским соглашением

http://www.mathnet.ru/rus/agreement

Параметры загрузки:

IP : 3.81 .55 .215

26 апреля 2023 г., 12:51:57 
УДК: 519.212.2+519.214.5

\title{
Структурно эквивалентные цепочки в равновероятной полиномиальной схеме
}

\author{
А. М. Шойтов \\ Академия криптографии Российской Федерации, Москва
}

Получено 20.V.2011

Для последовательности $X_{1}, \ldots, X_{n}$ независимых случайных величин, имеющих равновероятное распределение на множестве $\{1, \ldots, N\}$, получено описание предельных дискретных распределений чисел наборов из $k$ структурно эквивалентных $s$-цепочек при $N, n, s \rightarrow \infty, s N^{-1} \rightarrow \alpha \in(0,1)$, $n(N)_{s} N^{-s} \rightarrow \lambda \in(0, \infty)$ и произвольном $k \geqslant 2$. Доказательства используют метод Чена - Стейна.

Ключевые слова: последовательности независимых испытаний, равновероятная полиномиальная схема, структурно эквивалентные цепочки, метод Чена-Стейна

Structurally equivalent tuples in the equiprobable polynomial scheme

\section{A. M. Shoitov}

Academy of Cryptography of the Russian Federation, Moscow

Abstract. Let $X_{1}, \ldots, X_{n}$ be a sequence of independent random variables with the uniform distribution on the set $\{1, \ldots, N\}$. We describe limit discrete distributions of the number of $k$-element sets consisting of structurally equivalent $s$-tuples for $N, n, s \rightarrow \infty, s N^{-1} \rightarrow$ $\rightarrow \alpha \in(0,1), n(N)_{s} N^{-s} \rightarrow \lambda \in(0, \infty)$ and arbitrary $k \geqslant 2$. The proofs are based on the Chen - Stein method.

Key words: sequences of independent trials, equiprobable polynomial scheme, structurally equivalent $s$-tuples, Chen-Stein method

Citation: Mathematical Aspects of Cryptography, 2012, vol. 3, no. 3, pp. 129-151 (Russian). 


\section{§1. Введение и основные обозначения}

Для произвольного натурального $s$ две цепочки $\left(a_{1}, \ldots, a_{s}\right)$ и $\left(b_{1}, \ldots, b_{s}\right)$ символов алфавита $A=\{1, \ldots, N\}$ называются структурно эквивалентными (см. [6]) $\left(\left(a_{1}, \ldots, a_{s}\right) S_{N}\left(b_{1}, \ldots, b_{s}\right)\right)$, если существует такая подстановка $h$ на множестве символов алфавита $A$, при которой

$$
b_{i}=h\left(a_{i}\right), \quad i=1, \ldots, s .
$$

Под структурой цепочки понимается расположение пар одинаковых и разных символов в ней. Другими словами, две цепочки $\left(a_{1}, \ldots, a_{s}\right)$ и $\left(b_{1}, \ldots, b_{s}\right)$ обладают одинаковой структурой (пишут $\left.S\left(\left(a_{1}, \ldots, a_{s}\right)\right)=S\left(\left(b_{1}, \ldots, b_{s}\right)\right)\right)$ тогда и только тогда, когда

$$
a_{i}=a_{j} \Longleftrightarrow b_{i}=b_{j}, \quad 1 \leq i<j \leq s .
$$

Очевидно, что структурно эквивалентными являются цепочки с одинаковой структурой и только они. Отметим, что отношение структурной эквивалентности является частным случаем Н-эквивалентности (см. монографию [1]).

Структурная эквивалентность цепочек является одним из интересных с точки зрения криптографических приложений видов зависимости. Наличие в псевдослучайной последовательности повышенного числа цепочек с одинаковой структурой снижает ее криптографическое качество. В связи с этим представляется актуальной задача изучения предельных распределений случайных величин, характеризующих структурную эквивалентность цепочек в случайных последовательностях.

Пусть $\mathbf{X}=\left\{X_{1}, \ldots, X_{n}, \ldots\right\}$ - случайная последовательность, полученная по равновероятной полиномиальной схеме испытаний с исходами в множестве $A=\{1, \ldots, N\}$. Положим $Y_{i}(s)=\left(X_{i}, \ldots, X_{i+s-1}\right), i=1,2, \ldots, n$, и для произвольного натурального $k, k \geq 2$, определим случайные величины

$$
\xi_{n, k}=\sum_{1 \leq i_{1}<i_{2}<\ldots<i_{k} \leq n} \mathbf{I}\left\{S\left(Y_{i_{1}}(s)\right)=S\left(Y_{i_{2}}(s)\right)=\ldots=S\left(Y_{i_{k}}(s)\right)\right\},
$$

равные числу наборов из $k$ структурно эквивалентных $s$-цепочек в отрезке последовательности $\mathbf{X}$ длины $n+s-1$. Асимптотическое поведение случайных величин $\xi_{n, k}$ будем исследовать в схеме серий при $n \rightarrow \infty$, для которой параметры $s$ и $N$ могут меняться, а параметр $k$ фиксирован. Кроме того, вместо $\xi_{n, 2}$ будем также писать $\xi_{n}$. 
Достаточные условия сходимости распределения случайной величины $\xi_{n}$ к закону Пуассона в равновероятной схеме впервые были получены С. М. Буравлевым в работе [2]. Было предпринято немало попыток обобщения результатов работы [2] и ослабления соответствующих достаточных условий (см. $[4,6,8])$. Наилучшие достаточные условия сходимости распределения случайной величины $\xi_{n}$ к закону Пуассона в равновероятной схеме найдены в работе [9]. В ней была доказана следующая теорема.

Теорема 1 ([9]). Если при $n \rightarrow \infty$ параметры $N, s$ стремятся к бесконечности так, что $\mathbf{E} \xi_{n} \rightarrow \lambda, 0<\lambda<\infty$, и для некоторого числа $\epsilon, 0<\epsilon<1$, при достаточно больших $n$ выполнено условие

$$
s N^{-1} \geq 1-s^{-\epsilon},
$$

mo

$$
\lim _{n \rightarrow \infty} \mathbf{P}\left\{\xi_{n}=m\right\}=\frac{\lambda^{m}}{m !} e^{-\lambda}, \quad m=0,1, \ldots
$$

ЗАМЕЧАНИЕ 1. В работе [2] для сходимости распределения $\xi_{n}$ к закону Пуассона требуется выполнение условия $s N^{-1} \geq \ln N+1$, при котором с вероятностью, стремящейся к 1 , в $Y_{1}(s), \ldots, Y_{n}(s)$ отсутствуют $s$-цепочки, содержащие не все символы алфавита $A$. При условии $s N^{-1}-\frac{1}{4} \ln N \rightarrow$ $\rightarrow \infty$ работы [4] в $Y_{1}(s), \ldots, Y_{n}(s)$ с отделенной от 0 вероятностью существуют $s$-цепочки, не содержащие отдельных знаков. В свою очередь, при условии (1) в $Y_{1}(s), \ldots, Y_{n}(s)$ с отделенной от 0 вероятностью существуют $s$-цепочки, которые не содержат долю знаков алфавита $A$, пропорциональную его мощности $N$.

Из теоремы 2, доказываемой ниже, следует, что условие (1) близко к необходимому условию для сходимости $\xi_{n}$ к закону Пуассона в следующем смысле. Если при $n \rightarrow \infty$ параметры $N, s$ меняются так, что $s, N \rightarrow \infty$ и выполнены условия

$$
\begin{gathered}
s N^{-1} \rightarrow \alpha, \quad 0<\alpha<1, \\
n(N)_{s} N^{-s} \rightarrow \lambda, \quad 0<\lambda<\infty,
\end{gathered}
$$

то распределение случайной величины $\xi_{n}$ сходится к свертке двух дискретных распределений пуассоновского типа.

В настоящей работе дано описание асимптотического поведения случайных величин $\xi_{n, k}$ в равновероятной схеме при выполнении условий (2) и (3) для произвольного фиксированного натурального $k, k \geq 2$. Для 
доказательства используется функциональная версия метода Чена-Стейна (см. [10]), которая, по мнению автора, в настоящее время является одним из мощных инструментов доказательства предельных теорем пуассоновского типа в задачах вероятностной комбинаторики.

\section{§2. Основные результаты}

Обозначим через $\xi$ и $\eta$ две независимые случайные величины, распределеные по сложному закону Пуассона каждая с производящими функциями

$$
\begin{gathered}
\mathbf{E} z^{\xi}=\exp \left\{\frac{\lambda_{1}(z-1)}{1-(1-\alpha)^{2} z}\right\}, \quad \lambda_{1}=\alpha(2-\alpha) \frac{\lambda^{2}}{2}\left(e^{\frac{\alpha^{2}}{2(1-\alpha)^{2}}}-1\right), \\
\mathbf{E} z^{\eta}=\exp \left\{\frac{\lambda_{2}(z-1)}{1-(1-\alpha) z}\right\}, \quad \lambda_{2}=\alpha \lambda .
\end{gathered}
$$

Через $\mathcal{L}(Z)$ обозначим распределение случайной величины $Z$.

Теорема 2. Пусть при $n \rightarrow \infty$ параметры $N, s$ меняются так, что $s, N \rightarrow \infty$ и выполнены условия (2) $u$ (3). Тогда:

a) $\mathcal{L}\left(\xi_{n}\right) \rightarrow \mathcal{L}\left(\xi+C_{\eta}^{2}\right)$;

б) $\mathcal{L}\left(\xi_{n, k}\right) \rightarrow \mathcal{L}\left(C_{\eta}^{k}\right), k \geq 3$.

Формулировка п. а) теоремы 2, анонсированная автором в [9], содержала описку.

Пусть $\mu(a)$ - число различных символов в цепочке $a, a \in A^{r}, r=$ $=1,2, \ldots$ Определим случайные величины:

$$
\begin{gathered}
\eta_{n}=\sum_{i=1}^{n} \mathbf{I}\left\{\mu\left(Y_{i}(s)\right)=s\right\}, \\
\xi_{n, k}^{(1)}=\sum_{1 \leq i_{1}<i_{2}<\ldots<i_{k} \leq n} \mathbf{I}\left\{S\left(Y_{i_{1}}(s)\right)=S\left(Y_{i_{2}}(s)\right)=\ldots=S\left(Y_{i_{k}}(s)\right), \mu\left(Y_{i_{1}}(s)<s\right\},\right. \\
\xi_{n, k}^{(0)}=\sum_{1 \leq i_{1}<i_{2}<\ldots<i_{k} \leq n} \mathbf{I}\left\{S\left(Y_{i_{1}}(s)\right)=S\left(Y_{i_{2}}(s)\right)=\ldots=S\left(Y_{i_{k}}(s)\right), \mu\left(Y_{i_{1}}(s)=s\right\} .\right.
\end{gathered}
$$

По определению положим $\xi_{n}^{(i)}=\xi_{n, 2}^{(i)}, \quad i=0,1$. 
Теорема 3. Пусть при $n \rightarrow \infty$ параметры $N, s$ меняются так, что $s, N \rightarrow \infty$ и выполнены условия (2) $u$ (3). Тогда:

a) $\mathcal{L}\left(\left(\xi_{n}^{(1)}, \eta_{n}\right)\right) \rightarrow \mathcal{L}((\xi, \eta))$;

б) $\xi_{n, k}^{(1)} \rightarrow 0$ по вероятности, $k \geq 3$.

ЗАМЕчание 2. Ясно, что при $k \geq 2$

$$
\xi_{n, k}=\xi_{n, k}^{(0)}+\xi_{n, k}^{(1)} .
$$

Кроме того, очевидно, что

$\left\{S\left(Y_{i_{1}}(s)\right)=\ldots=S\left(Y_{i_{k}}(s)\right), \mu\left(Y_{i_{1}}(s)\right)=s\right\}=\left\{\mu\left(Y_{i_{r}}(s)\right)=s, r=1, \ldots, k\right\}$,

следовательно, $\xi_{n, k}^{(0)}=C_{\eta_{n}}^{k}$.

ЗАМЕчАнИЕ 3. Из теоремы 3 следует теорема 2. Также при условиях этих теорем имеют место соотношения

$$
\mathcal{L}\left(\xi_{n}^{(1)}\right) \rightarrow \mathcal{L}(\xi), \mathcal{L}\left(\eta_{n}\right) \rightarrow \mathcal{L}(\eta)
$$

ЗАМЕчАНИЕ 4. Нетрудно проверить, что при $s=o(N)$ в случае ограниченного математического ожидания случайной величины $\xi_{n}$ еe дисперсия стремится к бесконечности и $\mathbf{E} \xi_{n}^{(1)}=o\left(\mathbf{E} \xi_{n}^{(0)}\right)$. В этом случае оценки вероятности $\mathbf{P}\left\{\xi_{n}^{(1)}>0\right\}$ удалось получить В. Г. Михайлову в статье [5].

\section{§3. Доказательства}

В доказательствах для оценки точности пуассоновской аппроксимации распределения сумм зависимых индикаторов используется функциональный вариант метода Чена-Стейна (см. теорему 10.А в [10]).

С учетом замечания 2 доказательство разобьем на три части.

Сначала построим более просто исследуемые случайные величины $\widehat{\eta}_{n}$ и $\widehat{\xi}_{n}^{(12)}$, для которых при $n \rightarrow \infty$ справедливы оценки

$$
\begin{gathered}
\mathbf{P}\left\{\widehat{\eta}_{n} \neq \eta_{n}\right\}=o(1), \\
\mathbf{P}\left\{\widehat{\xi}_{n}^{(12)} \neq \xi_{n}^{(1)}\right\}=o(1) .
\end{gathered}
$$

Затем докажем, что случайные величины $\widehat{\eta}_{n}$ и $\widehat{\xi}_{n}^{(12)}$ асимптотически независимы и сходятся к случайным величинам $\eta$ и $\xi$ соответственно; отсюда будут следовать утверждения пп. а) теорем 2 и 3. 
И, наконец, докажем пп. б) теорем 2 и 3.

1. С учетом (2) и (3) нетрудно видеть, что при $n \rightarrow \infty$ справедливы оценки

$$
\begin{gathered}
\mathbf{E} \eta_{n}=n(N)_{s} N^{-s} \rightarrow \lambda, \quad 0<\lambda<\infty, \\
\mathbf{E} \xi_{n}^{(0)}=\sum_{r=1}^{s-1}(n-r)(N)_{s}(N-s+r)_{r} N^{-s-r}+C_{n-s+1}^{2}\left((N)_{s} N^{-s}\right)^{2}= \\
=\left(\lambda\left(\alpha^{-1}-1\right)+\frac{\lambda^{2}}{2}\right)(1+o(1)),
\end{gathered}
$$

из которых, в частности, следует, что

$$
N^{r}=o(n)
$$

для любого фиксированного натурального $r$.

Для натурального $i, N \leq i \leq n-N$, определим события:

$$
\begin{gathered}
A_{i}^{(1)}=\left\{\exists i_{1}, i_{2}, \quad i-\left[N^{1 / 3}\right] \leq i_{1} \leq i-1,\right. \\
\left.i+s-\left[N^{1 / 3}\right] \leq i_{2} \leq i+s-1: X_{i_{1}}=X_{i_{2}}\right\}, \\
A_{i}^{(2)}=\left\{\exists i_{1}, i_{2}, \quad i \leq i_{1} \leq i+\left[N^{1 / 3}\right]-1,\right. \\
\left.i+s \leq i_{2} \leq i+s+\left[N^{1 / 3}\right]-1: X_{i_{1}}=X_{i_{2}}\right\}, \\
B_{i}^{(1)}=\left\{\exists j_{1}, j_{2}, \quad i-\left[N^{1 / 3}\right] \leq j_{1}<j_{2} \leq i-1: \quad X_{j_{1}}=X_{j_{2}}\right\}, \\
B_{i}^{(2)}=\left\{\exists j_{1}, j_{2}, \quad i+s \leq j_{1}<j_{2} \leq i+s+\left[N^{1 / 3}\right]-1: X_{j_{1}}=X_{j_{2}}\right\}, \\
C_{i}=\left\{\exists l_{1}, l_{2}, \quad i-\left[N^{1 / 3}\right] \leq l_{1} \leq i-1<l_{2} \leq i+[s / 2]-1: X_{l_{1}}=X_{l_{2}}\right\}, \\
D_{i}=\left\{\mu\left(Y_{i-[s / 2]}([s / 2])\right)=[s / 2]\right\} .
\end{gathered}
$$

Рассмотрим семейство случайных индикаторов:

$$
\begin{gathered}
\Gamma_{0}=\left\{\gamma_{i, r}^{(0)}: i=N+1, \ldots, n-N, r=1, \ldots,\left[N^{1 / 3}\right]\right\}, \\
\gamma_{i, r}^{(0)}=\mathbf{I}\left\{\theta_{i-1}=0, \theta_{i}=\theta_{i+1}=\ldots=\theta_{i+r-1}=1, \theta_{i+r}=0\right\}, \\
\theta_{i}=\mathbf{I}\left\{\mu\left(Y_{i}(s)\right)=s, \bar{A}_{i}^{(1)}, \bar{A}_{i}^{(2)}, \bar{B}_{i}^{(1)}, \bar{B}_{i}^{(2)}, C_{i}, \bar{D}_{i}\right\}, \quad i=N, \ldots, n-N .
\end{gathered}
$$


Определим случайную величину:

$$
\widehat{\eta}_{n}=\sum_{r=1}^{\left[N^{1 / 3}\right]} \sum_{i=N+1}^{n-N} r \gamma_{i, r}^{(0)} .
$$

Из определения случайных величин $\theta_{i}, i=N, \ldots, n-N$, следует, что условие $\theta_{i}=\theta_{i+1}=\theta_{i+r-1}=1$ в определении $\gamma_{i, r}^{(0)}$ влечет за собой равенство

$$
\mu\left(Y_{i}(s+r-1)\right)=s+r-1 .
$$

Лемма 1. В условиях теорем 2 и 3 справедлива оиенка

$$
\mathbf{P}\left\{\widehat{\eta}_{n} \neq \eta_{n}\right\}=o(1) .
$$

Определим случайную величину

$$
\widetilde{\eta}_{n}=\sum_{i=N}^{n-N} \theta_{i} .
$$

Нетрудно видеть, что

$$
\begin{gathered}
\mathbf{P}\left\{\mu\left(Y_{i}(s)\right)=s, A_{i}^{(j)}\right\} \leq(N)_{s} N^{-s}\left(1-\left(\frac{N-\left[N^{1 / 3}\right]}{N}\right)^{\left[N^{1 / 3}\right]}\right), \quad j=1,2, \\
\mathbf{P}\left\{\mu\left(Y_{i}(s)\right)=s, B_{i}^{(j)}\right\} \leq(N)_{s} N^{-s}\left(1-(N)_{\left[N^{1 / 3}\right]} N^{-\left[N^{1 / 3}\right]}\right), \quad j=1,2, \\
\mathbf{P}\left\{\mu\left(Y_{i}(s)\right)=s, \overline{C_{i}}\right\} \leq(N)_{s} N^{-s}\left(\frac{[s / 2]}{N}\right)^{\left[N^{1 / 3}\right]}, \\
\mathbf{P}\left\{\mu\left(Y_{i}(s)\right)=s, D_{i}\right\} \leq(N)_{s} N^{-s}(N)_{[s / 2]} N^{-[s / 2]} .
\end{gathered}
$$

Тогда

$$
\begin{aligned}
\mathbf{P}\left\{\eta_{n} \neq\right. & \left.\widetilde{\eta}_{n}\right\} \leq \mathbf{E} \eta_{n}-\mathbf{E} \widetilde{\eta}_{n} \leq \sum_{i=1}^{N} \mathbf{I}\left\{\mu\left(Y_{i}(s)\right)=s\right\}+\sum_{i=n-N}^{n} \mathbf{I}\left\{\mu\left(Y_{i}(s)\right)=s\right\}+ \\
& +\sum_{i=N}^{n-N} \mathbf{P}\left\{\mu\left(Y_{i}(s)\right)=s, A_{i}^{(1)}\right\}+\sum_{i=N}^{n-N} \mathbf{P}\left\{\mu\left(Y_{i}(s)\right)=s, A_{i}^{(2)}\right\}+ \\
& +\sum_{i=N}^{n-N} \mathbf{P}\left\{\mu\left(Y_{i}(s)\right)=s, B_{i}^{(1)}\right\}+\sum_{i=N}^{n-N} \mathbf{P}\left\{\mu\left(Y_{i}(s)\right)=s, B_{i}^{(2)}\right\}+
\end{aligned}
$$

2012. T. 3. № 3. C. 129-151 


$$
\begin{gathered}
+\sum_{i=N}^{n-N} \mathbf{P}\left\{\mu\left(Y_{i}(s)\right)=s, \overline{C_{i}}\right\}+\sum_{i=N}^{n-N} \mathbf{P}\left\{\mu\left(Y_{i}(s)\right)=s, D_{i}\right\} \leq \\
\leq 2 N(N)_{s} N^{-s}+n(N)_{s} N^{-s}\left(2\left(1-\left(\frac{N-\left[N^{1 / 3}\right]}{N}\right)^{\left[N^{1 / 3}\right]}\right)+\right. \\
\left.+2\left(1-(N)_{\left[N^{1 / 3}\right]} N^{-\left[N^{1 / 3}\right]}\right)+\left(\frac{[s / 2]}{N}\right)^{\left[N^{1 / 3}\right]}+\left((N)_{[s / 2]} N^{-[s / 2]}\right)\right)=o(1) .
\end{gathered}
$$

Следовательно, предельные распределения $\eta_{n}$ и $\widetilde{\eta}_{n}$ совпадают.

Далее, из определений $\widehat{\eta}_{n}$ и $\widetilde{\eta}_{n}$ следует, что $\widehat{\eta}_{n} \leq \widetilde{\eta}_{n}$. Нетрудно проверить, что

$$
\mathbf{E} \widehat{\eta}_{n}=n(N)_{s} N^{-s}(1+o(1)),
$$

и, следовательно,

$$
\mathbf{P}\left\{\widehat{\eta}_{n} \neq \widetilde{\eta}_{n}\right\} \leq \mathbf{E} \widetilde{\eta}_{n}-\mathbf{E} \widehat{\eta}_{n}=o(1) .
$$

Лемма 1 доказана.

Перейдем к изучению асимптотического поведения случайной величины $\xi_{n}^{(1)}$. Положим

$$
\begin{gathered}
\xi_{n}^{(12)}=\sum_{1 \leq i_{1}<i_{1}+s \leq i_{2} \leq n} \mathbf{I}\left\{S\left(Y_{i_{1}}(s)\right)=S\left(Y_{i_{2}}(s)\right), \mu\left(Y_{i_{2}}(s)\right)<s\right\}, \\
\xi_{n}^{(13)}=\sum_{1 \leq i_{1}<i_{2} \leq n, i_{2}-i_{1} \leq s-1} \mathbf{I}\left\{S\left(Y_{i_{1}}(s)\right)=S\left(Y_{i_{2}}(s)\right), \mu\left(Y_{i_{2}}(s)\right)<s\right\} .
\end{gathered}
$$

Очевидно, что

$$
\xi_{n}^{(1)}=\xi_{n}^{(12)}+\xi_{n}^{(13)} .
$$

С учетом доказательства теоремы 3 статьи [9] нетрудно видеть, что

$$
\begin{gathered}
\mathbf{E} \xi_{n}^{(12)}=C_{n}^{2} N^{-2 s} \sum_{m=1}^{s-1} \sigma(s, m)\left((N)_{m}\right)^{2}(1+o(1)), \\
\mathbf{E} \xi_{n}^{(13)}=\sum_{r=1}^{s-1}(n-r) \mathbf{P}\left\{S\left(Y_{1}(s)\right)=S\left(Y_{1+r}(s)\right), \mu\left(Y_{1}(s)\right)<s\right\} .
\end{gathered}
$$

Лемма 2. В условиях теорем 2 и 3 справедлива оценка

$$
\mathbf{E} \xi_{n}^{(13)}=o(1) .
$$


Доказательство. Рассмотрим $r$-е слагаемое в выражении (9) для $\mathbf{E} \xi_{n}^{(13)}$. Фиксируем произвольно символы цепочки $Y_{1}(r)$. Символ $X_{1+r}$ можно выбрать не более чем $N$ вариантами. Из структурной эквивалентности цепочек $Y_{1}(s)$ и $Y_{1+r}(s)$ следует, что если $X_{1}=X_{2}$, то $X_{2+r}=X_{1+r}$, в противном случае имеем для $X_{2+r}$ ровно $N-1$ возможных вариантов, и т.д. Последовательно определяя все символы цепочки $Y_{1+r}(s)$, убеждаемся в том, что количество таких цепочек не превосходит величины $(N)_{q\left(\mu\left(Y_{1}(r)\right)\right)}$, где

$$
q\left(\mu\left(Y_{1}(r)\right)\right)=\min \left\{s-1, s-\left(r-\mu\left(Y_{1}(r)\right)\right)\right\}
$$

следовательно,

$$
\mathbf{P}\left\{S\left(Y_{1}(s)\right)=S\left(Y_{1+r}(s)\right), \mu\left(Y_{1}(s)\right)<s \mid \mu\left(Y_{1}(r)=\mu\right\} \leq(N)_{q(\mu)} N^{-s} .\right.
$$

Далее:

$$
\begin{gathered}
\mathbf{E} \xi_{n}^{(13)}=\sum_{r=1}^{\left[s^{2 / 3}\right]}(n-r) \mathbf{P}\left\{S\left(Y_{1}(s)\right)=S\left(Y_{1+r}(s)\right), \mu\left(Y_{1}(s)\right)<s\right\}+ \\
+\sum_{r=\left[s^{2 / 3}\right]+1}^{s-1}(n-r) \mathbf{P}\left\{S\left(Y_{1}(s)\right)=S\left(Y_{1+r}(s)\right), \mu\left(Y_{1}(s)\right)<s \mid \mu\left(Y_{1}(r)\right) \leq r-2\right\} \times \\
\times \mathbf{P}\left\{\mu\left(Y_{1}(r)\right) \leq r-2\right\}+ \\
+\sum_{r=\left[s^{2 / 3}\right]+1}^{s-1}(n-r) \mathbf{P}\left\{S\left(Y_{1}(s)\right)=S\left(Y_{1+r}(s)\right), \mu\left(Y_{1}(s)\right)<s \mid \mu\left(Y_{1}(r)\right) \geq r-1\right\} \times \\
\times \mathbf{P}\left\{\mu\left(Y_{1}(r)\right) \geq r-1\right\} .
\end{gathered}
$$

С учетом оценок (2), (3), (7) и неравенства (11) получаем оценки:

$$
\begin{gathered}
\sum_{r=1}^{\left[s^{2 / 3}\right]}(n-r) \mathbf{P}\left\{S\left(Y_{1}(s)\right)=S\left(Y_{1+r}(s)\right), \mu\left(Y_{1}(s)\right)<s\right\} \leq \\
\leq n \sum_{r=1}^{\left[s^{2 / 3}\right]}(N)_{s-1} N^{-s} \leq n s^{2 / 3}(N)_{s-1} N^{-s}=O\left(s^{-\frac{1}{3}}\right), \\
\sum_{r=\left[s^{2 / 3}\right]+1}^{s-1}(n-r) \mathbf{P}\left\{S\left(Y_{1}(s)\right)=S\left(Y_{1+r}(s)\right), \mu\left(Y_{1}(s)\right)<s \mid \mu\left(Y_{1}(r)\right) \leq r-2\right\} \times
\end{gathered}
$$




$$
\begin{gathered}
\times \mathbf{P}\left\{\mu\left(Y_{1}(r)\right) \leq r-2\right\} \leq \\
\leq n \sum_{r=\left[s^{2 / 3}\right]+1}^{s-1}(N)_{s-2} N^{-s} \mathbf{P}\left\{\mu\left(Y_{1}(r)\right) \leq r-2\right\} \leq n s(N)_{s-2} N^{-s}=O\left(s^{-1}\right), \\
\sum_{r=\left[s^{2 / 3}\right]+1}^{s-1}(n-r) \mathbf{P}\left\{S\left(Y_{1}(s)\right)=S\left(Y_{1+r}(s)\right), \mu\left(Y_{1}(s)\right)<s \mid \mu\left(Y_{1}(r)\right) \geq r-1\right\} \times \\
\times \mathbf{P}\left\{\mu\left(Y_{1}(r)\right) \geq r-1\right\} \leq \\
\leq n \sum_{r=\left[s^{2 / 3}\right]+1}^{s-1}(N)_{s-1} N^{-s} \mathbf{P}\left\{\mu\left(Y_{1}(r)\right) \geq r-1\right\}=O\left(s^{4 / 3} e^{-\frac{1}{2} s^{-1 / 3}}\right) .
\end{gathered}
$$

Лемма 2 доказана.

Из леммы 2 следует, что случайную величину $\xi_{n}^{(13)}$ можно исключить из рассмотрения.

Далее исследуем асимптотическое поведение случайной величины $\xi_{n}^{(12)}$.

Лемма 3. В условиях теорем 2 и 3 справедлива оценка

$$
\mathbf{E} \xi_{n}^{(12)}=\frac{1}{2} \lambda^{2}\left(e^{\frac{\alpha^{2}}{2(1-\alpha)^{2}}}-1\right)(1+o(1))
$$

Доказательство. Пусть $\left\{t_{1}, \ldots, t_{n}, \ldots\right\}$ - такая последовательность натуральных чисел, что $t_{n} \rightarrow \infty$ при $n \rightarrow \infty$. Тогда из оценки (9) следует, что

$$
\mathbf{E} \xi_{n}^{(12)}=C_{n}^{2} N^{-2 s}\left(\sum_{m=1}^{s-t_{n}} \sigma(s, m)\left((N)_{m}\right)^{2}+\sum_{m=s-t_{n}+1}^{s-1} \sigma(s, m)\left((N)_{m}\right)^{2}\right)(1+o(1)) .
$$

Далее, из известного неравенства

$$
\sigma(s, m-1) \leq \sigma(s, m) \frac{C_{m}^{2}}{s-m+1}, \quad 2 \leq m \leq s,
$$

следует, что для $1 \leq t \leq s-1$

$$
\begin{gathered}
\sigma(s, s-t)\left((N)_{s-t}\right)^{2}\left(\sigma(s, s-t+1)\left((N)_{s-t+1}\right)^{2}\right)^{-1} \leq \\
\leq \frac{C_{s-t+1}^{2}}{t(N-s+t)^{2}}<\frac{s^{2}}{2 t(N-s)^{2}} .
\end{gathered}
$$


Следовательно, при $n \rightarrow \infty$

$$
C_{n}^{2} N^{-2 s} \sum_{m=1}^{s-t_{n}} \sigma(s, m)\left((N)_{m}\right)^{2} \rightarrow 0
$$

Далее,

$$
C_{n}^{2} N^{-2 s} \sum_{m=s-t_{n}+1}^{s-1} \sigma(s, m)\left((N)_{m}\right)^{2}=C_{n}^{2} N^{-2 s} \sum_{t=1}^{t_{n}-1} \sigma(s, s-t)\left((N)_{s-t}\right)^{2} .
$$

Из замечания к теореме 1 работы [3] следует, что при $s \rightarrow \infty$ и $t=o\left(s^{1 / 2}\right)$ справедлива оценка

$$
\sigma(s, s-t)=C_{s}^{t}\left(\frac{s-t}{2}\right)^{t}\left(1+O\left(t s^{-1 / 2}\right)\right)
$$

из которой с учетом формулы Стирлинга вытекает, что

$$
\begin{gathered}
N^{-2 s} \sigma(s, s-t)\left((N)_{s-t}\right)^{2}= \\
=N^{-2 s}\left((N)_{s}\right)^{2} C_{s}^{t}\left(\frac{s-t}{2}\right)^{t}\left(\frac{(N-s) !}{(N-s+t) !}\right)^{2}\left(1+O\left(t s^{-1 / 2}\right)\right)= \\
=N^{-2 s}\left((N)_{s}\right)^{2}\left(\frac{s^{2}}{2(N-s)^{2}}\right)^{t} \frac{1}{t !}\left(1+O\left(t s^{-1 / 2}\right)\right) .
\end{gathered}
$$

Из того, что ряд $\sum_{t=1}^{\infty} \frac{q^{t}}{t !}$ сходится для любого действительного $q$, и из оценок (13), (15) и (16) следует, что

$$
\begin{gathered}
\mathbf{E} \xi_{n}^{(12)}=\frac{1}{2} \lambda^{2}(1+o(1)) \sum_{t=1}^{\infty}\left(\frac{s^{2}}{2(N-s)^{2}}\right)^{t} \frac{1}{t !}= \\
=\frac{1}{2} \lambda^{2}\left(e^{\frac{s^{2}}{2(N-s)^{2}}}-1\right)(1+o(1))=\frac{1}{2} \lambda^{2}\left(e^{\frac{\alpha^{2}}{2(1-\alpha)^{2}}}-1\right)(1+o(1)) .
\end{gathered}
$$

Лемма 3 доказана.

Из (12) и (6) также вытекает оценки:

$$
\begin{gathered}
\sum_{1 \leq i_{1}<i_{1}+s \leq i_{2} \leq n} \mathbf{P}\left\{S\left(Y_{i_{1}}(s)\right)=S\left(Y_{i_{2}}(s)\right)\right\}=\frac{1}{2} \lambda^{2} e^{\frac{\alpha^{2}}{2(1-\alpha)^{2}}}(1+o(1)), \\
\mathbf{E} \xi_{n}=\left(\frac{1}{2} \lambda^{2} e^{\frac{\alpha^{2}}{2(1-\alpha)^{2}}}+\lambda\left(\alpha^{-1}-1\right)\right)(1+o(1)) .
\end{gathered}
$$


Кроме того, из (14) следует, что число совпадающих символов для пары неперекрывающихся структурно эквивалентных цепочек ограничено по вероятности.

Пусть $\left\{t_{1}, \ldots, t_{n}, \ldots\right\}$ и $\left\{T_{1}, \ldots, T_{n}, \ldots\right\}$ - две такие неубывающие последовательности натуральных чисел, что $T_{n} t_{n}=o(s)$ при $n \rightarrow \infty$. Тогда из (15) несложно выводится, что равномерно по $t<t_{n}$

$$
\begin{gathered}
\sigma(s, s-t)\left((N)_{s-t}\right)^{2}= \\
=\sigma\left(s-T_{n}, s-T_{n}-t\right)\left((N)_{s-T_{n}-t}\right)^{2}\left(\left(N-s+T_{n}+t\right)_{T_{n}}\right)^{2}\left(1+O\left(T_{n} t_{n} s^{-1}\right)\right) .
\end{gathered}
$$

Следовательно, для двух неперекрывающихся структурно эквивалентных цепочек вероятность того, что на заданном отрезке цепочки длины $O(s)$ не содержится одинаковых символов и среди остальных символов цепочки не встретилось символов, лежащих на этом отрезке, стремится к единице.

Далее положим $T_{n}=\left[s^{5 / 6}\right], t_{n}=\left[s^{1 / 7}\right]$, для натурального $i, 1 \leq i \leq n$, определим события:

$$
\begin{gathered}
F_{i}=\left\{\exists j_{1}, j_{2}, i \leq j_{1} \leq i+s-T_{n}<j_{2} \leq i+s-1: X_{j_{1}}=X_{j_{2}}\right\}, \\
G_{i}=\left\{\exists j_{1}, j_{2}, i+s-T_{n} \leq j_{1}<j_{2} \leq i+s-1: X_{j_{1}}=X_{j_{2}}\right\}, \\
H_{i}=\left\{\exists j_{1}, j_{2}, i \leq j_{1}<j_{2} \leq i+T_{n}-1: X_{j_{1}}=X_{j_{2}}\right\},
\end{gathered}
$$

и для $N \leq i_{1}<i_{1}+\left[n^{1 / 2}\right] \leq i_{2} \leq n-N$ рассмотрим семейство случайных индикаторов

$$
\begin{gathered}
\vartheta_{i_{1}, i_{2}}=\mathbf{I}\left\{S\left(Y_{i_{1}}(s)\right)=S\left(Y_{i_{2}}(s)\right), s-t_{n} \leq \mu\left(Y_{i_{2}}(s)\right) \leq s-1,\right. \\
\left.\bar{A}_{i_{2}}^{(1)}, \bar{A}_{i_{2}}^{(2)}, \bar{B}_{i_{2}}^{(1)}, \bar{B}_{i_{2}}^{(2)}, \bar{F}_{i_{2}}, \bar{G}_{i_{2}}, \bar{H}_{i_{2}}\right\},
\end{gathered}
$$

где события $A_{i}^{(1)}, A_{i}^{(2)}, B_{i}^{(1)}, B_{i}^{(2)}, N \leq i \leq n-N$, определены перед леммой 1 .

Рассмотрим семейство случайных индикаторов

$$
\Gamma_{1}=\left\{\gamma_{i_{1}, i_{2}, q}^{(1)}: N+1 \leq i_{1}<i_{1}+\left[n^{1 / 2}\right] \leq i_{2} \leq n-N, q=1, \ldots,\left[N^{1 / 3}\right]\right\},
$$

где

$$
\begin{aligned}
& \gamma_{i_{1}, i_{2}, q}^{(1)}= \\
& =\mathbf{I}\left\{\vartheta_{i_{1}, i_{2}}=\vartheta_{i_{1}+1, i_{2}+1}=\ldots=\vartheta_{i_{1}+q-1, i_{2}+q-1}=1, \vartheta_{i_{1}-1, i_{2}-1}=\vartheta_{i_{1}+q, i_{2}+q}=0\right\},
\end{aligned}
$$


и определим случайную величину

$$
\widehat{\xi}_{n}^{(12)}=\sum_{q=1}^{\left[N^{1 / 3}\right]} \sum_{N \leq i_{1}<i_{1}+\left[n^{1 / 2}\right] \leq i_{2} \leq n-N} q \gamma_{i_{1}, i_{2}, q}^{(1)} .
$$

Лемма 4. В условиях теорем 2 и 3 справедлива оценка

$$
\mathbf{P}\left\{\widehat{\xi}_{n}^{(12)} \neq \xi_{n}^{(12)}\right\}=o(1)
$$

Доказательство. Определим случайные величины

$$
\widetilde{\xi}_{n}^{(12)}=\sum_{N \leq i_{1}<i_{1}+\left[n^{1 / 2}\right] \leq i_{2} \leq n-N} \vartheta_{i_{1}, i_{2}} .
$$

По аналогии с оценкой для величины $\mathbf{E} \eta_{n}-\mathbf{E} \widetilde{\eta}_{n}$, полученной в лемме 1 , доказывается оценка

$$
\mathbf{E} \xi_{n}^{(12)}-\mathbf{E} \widetilde{\xi}_{n}^{(12)}=o(1) .
$$

Из определения случайных величин $\vartheta_{i_{1}, i_{2}}$ следует, что условие $\vartheta_{i_{1}, i_{2}}=$ $=\vartheta_{i_{1}+1, i_{2}+1}=\ldots=\vartheta_{i_{1}+q-1, i_{2}+q-1}=1$ в определении $\gamma_{i_{1}, i_{2}, q}^{(1)}$ влечет за собой условие

$$
\mu\left(Y_{i}(s+r-1)\right)=\mu\left(Y_{i+r-1}(s)\right)+r-1=\mu\left(Y_{i}(s)\right)+r-1 .
$$

Далее, из определений случайных величин $\widehat{\xi}_{n}^{(12)}$ и $\widetilde{\xi}_{n}^{(12)}$ следует, что $\widehat{\xi}_{n}^{(12)} \leq \widetilde{\xi}_{n}^{(12)}$. Кроме того, равномерно по $q=1, \ldots,\left[N^{1 / 3}\right]$ и по $N \leq i_{1}<$ $<i_{1}+\left[n^{1 / 2}\right] \leq i_{2} \leq n-N$ справедливы оценки

$$
\begin{aligned}
\mathbf{E} \gamma_{i_{1}, i_{2}, q}^{(1)}= & N^{-2(s+r+1)}(1+o(1)) \sum_{t=1}^{t_{n}} \sigma(s-q, s-q-t)\left((N)_{s-q-t}\right)^{2} \times \\
& \times\left((N-s+q+t)_{2 q}\right)^{2}\left(N^{2}-(N-s-q+t)^{2}\right)^{2}= \\
= & \left((1-\alpha)^{2}\right)^{-(q-1)}\left(1-(1-\alpha)^{2}\right)^{2}(1+o(1)) \sum_{m=s-t_{n}}^{s-1} \sigma(s, m)\left((N)_{m}\right)^{2},
\end{aligned}
$$

следовательно, с учетом леммы 3 , справедлива оценка.

$$
\mathbf{E} \widehat{\xi}_{n}^{(12)}=\mathbf{E} \xi_{n}^{(12)}(1+o(1))
$$

Лемма 4 доказана. 
2. Докажем пп. а) теорем 2 и 3. Воспользуемся теоремой 10.А книги [10]. Рассмотрим семейство случайных индикаторов $\Gamma=\Gamma_{0} \bigcup \Gamma_{1}$ и семейство $\Gamma^{\prime}=\Gamma_{0}^{\prime} \bigcup \Gamma_{1}^{\prime}$, которое состоит из независимых в совокупности пуассоновских случайных величин $g_{i, r}^{(0)}, g_{i_{1}, i_{2}, q}^{(1)}$ :

$$
\begin{gathered}
\Gamma_{0}^{\prime}=\left\{g_{i, r}^{(0)}: i=N+1, \ldots, n-N, r=1, \ldots,\left[N^{1 / 3}\right]\right\}, \\
\Gamma_{1}^{\prime}=\left\{g_{i_{1}, i_{2}, q}^{(1)}: N+1 \leq i_{1}<i_{1}+\left[n^{1 / 2}\right] \leq i_{2} \leq n-N, q=1, \ldots,\left[N^{1 / 3}\right]\right\}, \\
\mathbf{E} g_{i, r}^{(0)}=\mathbf{E} \gamma_{i, r}^{(0)}, \mathbf{E} g_{i_{1}, i_{2}, q}^{(1)}=\mathbf{E} \gamma_{i_{1}, i_{2}, q}^{(1)} .
\end{gathered}
$$

Построим оценку точности аппроксимации распределения семейства $\Gamma$ распределением семейства $\Gamma^{\prime}$. Положим

$$
\begin{gathered}
J=J^{(0)} \bigcup J^{(1)}, \quad J^{(0)}=\left\{(i, r): i=N+1, \ldots, n-N, r=1, \ldots,\left[N^{1 / 3}\right]\right\}, \\
J^{(1)}=\left\{\left(i_{1}, i_{2}, q\right): N+1 \leq i_{1}<i_{1}+\left[n^{1 / 2}\right] \leq i_{2} \leq n-N, q=1, \ldots,\left[N^{1 / 3}\right]\right\} .
\end{gathered}
$$

Для $\iota \in J$ определим множество $J(\iota)=J^{(0)}(\iota) \bigcup J^{(1)}(\iota) \subseteq J, J^{(0)}(\iota) \subseteq J^{(0)}$, $J^{(1)}(\iota) \subseteq J^{(1)}$, следующим образом:

1. Если $\iota=\left(i_{1}, r_{1}\right) \in J^{(0)}$, то

$$
\begin{gathered}
J^{(0)}(\iota)=\left\{\left(i_{2}, r_{2}\right) \in J^{(0)},\left(i_{2}, r_{2}\right) \neq\left(i_{1}, r_{1}\right): \text { отрезки }\left[i_{1}-1, i_{1}+s+r_{1}-1\right]\right. \\
\text { и } \left.\left[i_{2}-1, i_{2}+s+r_{2}-1\right] \text { пересекаются }\right\}, \\
J^{(1)}(\iota)=\left\{\left(j_{1}, j_{2}, q\right) \in J^{(1)}: \text { отрезок }\left[i_{1}-1, i_{1}+s+r_{1}-1\right]\right.
\end{gathered}
$$

пересекается с одним из отрезков $\left.\left[j_{1}-1, j_{1}+s+q\right],\left[j_{2}-1, j_{2}+s+q\right]\right\}$.

2. Если $\iota=\left(j_{1}, j_{2}, q_{1}\right) \in J^{(1)}$, то

$$
J^{(0)}(\iota)=\left\{\left(i_{1}, r\right) \in J^{(0)}: \text { отрезок }\left[i_{1}-1, i_{1}+s+r-1\right]\right.
$$

пересекается с одним из отрезков $\left.\left[j_{1}-1, j_{1}+s+q_{1}\right],\left[j_{2}-1, j_{2}+s+q_{1}\right]\right\}$,

$$
J^{(1)}(\iota)=\left\{\left(i_{1}, i_{2}, q_{2}\right) \in J^{(1)},\left(i_{1}, i_{2}, q_{2}\right) \neq\left(j_{1}, j_{2}, q_{1}\right):\right.
$$

хотя бы один из отрезков $\left[i_{1}-1, i_{1}+s+q_{2}\right],\left[j_{2}-1, j_{2}+s+q_{1}\right]$

пересекается хотя бы с одним из отрезков

$$
\left.\left[j_{1}-1, j_{1}+s+q_{1}\right],\left[j_{2}-1, j_{2}+s+q_{1}\right]\right\} .
$$


Через $J_{1}^{(1)}(\iota)$ обозначим подмножество множества $J^{(1)}(\iota)$, соответствующее пересечению ровно одной пары отрезков, а через $J_{2}^{(1)}(\iota)-$ пересечению двух пар.

Согласно теореме 10.А книги [10] для расстояния по вариации между распределением $Г$ и распределением $\Gamma^{\prime}$ справедлива оценка

$$
\rho\left(\mathcal{L}(\Gamma), \mathcal{L}\left(\Gamma^{\prime}\right)\right) \leq \sum_{\iota_{1} \in J}\left(\mathbf{E} g_{\iota_{1}}^{\prime}\left(\mathbf{E} g_{\iota_{1}}^{\prime}+\sum_{\iota_{2} \in J\left(\iota_{1}\right)} \mathbf{E} g_{\iota_{2}}^{\prime}\right)\right)+\sum_{\iota_{1} \in J} \sum_{\iota_{2} \in J\left(\iota_{1}\right)} \mathbf{E}\left(g_{\iota_{1}}^{\prime} g_{\iota_{2}}^{\prime}\right)
$$

где $g_{\iota}^{\prime}=g_{\iota}^{(1)}$ при $\iota \in J^{(1)}$ и $g_{\iota}^{\prime}=g_{\iota}^{(0)}$ при $\iota \in J^{(0)}$. Очевидно, для доказательства того, что $\rho\left(\mathcal{L}(\Gamma), \mathcal{L}\left(\Gamma^{\prime}\right)\right) \rightarrow 0$ при $n \rightarrow \infty$, достаточно доказать, что

$$
\sum_{\iota_{1} \in J^{(l)}}\left(\mathbf{E} g_{\iota_{1}}^{\prime}\left(\mathbf{E} g_{\iota_{1}}^{\prime}+\sum_{\iota_{2} \in J^{(m)}\left(\iota_{1}\right)} \mathbf{E} g_{\iota_{2}}^{\prime}\right)\right)+\sum_{\iota_{1} \in J^{(l)}} \sum_{\iota_{2} \in J^{(m)}\left(\iota_{1}\right)} \mathbf{E}\left(g_{\iota_{1}}^{\prime} g_{\iota_{2}}^{\prime}\right) \rightarrow 0
$$

при $l, m=0,1$. Так как $\sum_{r=1}^{\left[N^{1 / 3}\right]} \mathbf{E} \gamma_{i, r}^{(0)} \leq \mathbf{E} \theta_{i}$, то, используя формулу для $\mathbf{E} \theta_{i}$ и условия теоремы, получим

$$
\begin{gathered}
\sum_{\iota_{1} \in J^{(0)}}\left(\mathbf{E} g_{\iota_{1}}^{\prime} \sum_{\left.\iota_{2} \in J(0)\left(\iota_{1}\right) \bigcup\left\{\iota_{1}\right)\right\}} \mathbf{E} g_{\iota_{2}}^{\prime}\right) \leq \\
\leq \sum_{i=N+1}^{n-N}\left(\mathbf{E} \theta_{i} \sum_{j=i-s-\left[N^{1 / 3}\right]}^{i+s+\left[N^{1 / 3}\right]} \mathbf{E} \theta_{j}\right)=O\left(s n^{-1}\right)=o(1),
\end{gathered}
$$

здесь и далее при $j \notin\{N, \ldots, n-N\}$ полагаем $\theta_{j}=0$. Далее, аналогично:

$$
\begin{gathered}
\sum_{\iota_{1} \in J^{(1)}}\left(\mathbf{E} g_{\iota_{1}}^{\prime} \sum_{\left.\iota_{2} \in J^{(0)}\left(\iota_{1}\right) \cup\left\{\iota_{1}\right)\right\}} \mathbf{E} g_{\iota_{2}}^{\prime}\right)=O\left(s n^{-1}\right)=o(1), \\
\sum_{\iota_{1} \in J^{(0)}}\left(\mathbf{E} g_{\iota_{1}}^{\prime} \sum_{\left.\iota_{2} \in J^{(1)}\left(\iota_{1}\right) \cup\left\{\iota_{1}\right)\right\}} \mathbf{E} g_{\iota_{2}}^{\prime}\right)=O\left(s n^{-1}\right)=o(1), \\
\sum_{\iota_{1} \in J^{(1)}}\left(\mathbf{E} g_{\iota_{1}}^{\prime} \sum_{\left.\iota_{2} \in J^{(1)}\left(\iota_{1}\right) \bigcup\left\{\iota_{1}\right)\right\}} \mathbf{E} g_{\iota_{2}}^{\prime}\right)=\sum_{\iota_{1} \in J^{(1)}}\left(\mathbf{E} g_{\iota_{1}}^{\prime} \sum_{\left.\iota_{2} \in J_{2}^{(1)}(\iota) \bigcup\left\{\iota_{1}\right)\right\}} \mathbf{E} g_{\iota_{2}}^{\prime}\right)+ \\
+\sum_{\iota_{1} \in J^{(1)}}\left(\mathbf{E} g_{\iota_{1}}^{\prime} \sum_{\left.\iota_{2} \in J_{1}^{(1)}(\iota) \cup\left\{\iota_{1}\right)\right\}} \mathbf{E} g_{\iota_{2}}^{\prime}\right)=O\left(s^{2} n^{-2}\right)+O\left(s n^{-1}\right)=o(1) .
\end{gathered}
$$

Следовательно, первое слагаемое в правой части (17) стремится к нулю. Оценим второе слагаемое.

2012. T. 3. № 3. C. $129-151$ 
Из определения случайных величин $\gamma_{i, r}^{(0)}$ следует (см. (8)), что если $\left(i_{2}, r_{2}\right) \in J^{(0)}\left(i_{1}, r_{1}\right)$, то равенство $\gamma_{i_{1}, r_{1}}^{(0)} \gamma_{i_{2}, r_{2}}^{(0)}=1$ возможно только в случаях $i_{2}-1=i_{1}+s+r_{1}-1$ и $i_{1}-1=i_{2}+s+r_{2}-1$. Следовательно,

$$
\begin{aligned}
& \sum_{\iota_{1} \in J^{(0)}} \sum_{\iota_{2} \in J^{(0)}\left(\iota_{1}\right)} \mathbf{E}\left(g_{\iota_{1}}^{\prime} g_{\iota_{2}}^{\prime}\right) \leq \sum_{i=N+1}^{n-N}\left(\sum_{j=i-s-\left[N^{1 / 3}\right]}^{i-s-1} \mathbf{E}\left(\theta_{i} \theta_{j}\right)+\sum_{j=i+s+1}^{i+s+\left[N^{1 / 3}\right]} \mathbf{E}\left(\theta_{i} \theta_{j}\right)\right)= \\
& =\sum_{i=N+1}^{n-N}\left(\sum_{j=i-s-\left[N^{1 / 3}\right]}^{i-s-1} \mathbf{E} \theta_{i} \mathbf{E} \theta_{j}+\sum_{j=i+s+1}^{i+s+\left[N^{1 / 3}\right]} \mathbf{E} \theta_{i} \mathbf{E} \theta_{j}\right)=O\left(N^{1 / 3} n^{-1}\right)=o(1) .
\end{aligned}
$$

Рассмотрим случай $\iota_{1} \in J^{(1)}, \iota_{2} \in J^{(0)}\left(\iota_{1}\right)$ (в случае $\iota_{1} \in J^{(0)}, \iota_{2} \in J^{(1)}\left(\iota_{1}\right)$ оценки аналогичны):

$$
\begin{gathered}
\sum_{\iota_{1} \in J^{(1)}} \sum_{\iota_{2} \in J^{(0)}\left(\iota_{1}\right)} \mathbf{E}\left(g_{\iota_{1}}^{\prime} g_{\iota_{2}}^{\prime}\right) \leq \sum_{N \leq i_{1}<i_{1}+\left[n^{1 / 2}\right] \leq i_{2} \leq n-N}\left(\sum_{j=i_{1}-s-\left[N^{1 / 3}\right]}^{i_{1}+s+\left[N^{1 / 3}\right]} \mathbf{E}\left(\vartheta_{i_{1}, i_{2}} \theta_{j}\right)+\right. \\
\left.+\sum_{j=i_{2}-s-\left[N^{1 / 3}\right]}^{i_{2}+s+\left[N^{1 / 3}\right]} \mathbf{E}\left(\vartheta_{i_{1}, i_{2}} \theta_{j}\right)\right)=O\left(N^{1 / 3} C_{n}^{2}\left((N)_{s}\right)^{3} N^{-3 s}\right)+ \\
+O\left(C_{n}^{2} \sum_{j=0}^{s-1} \mathbf{E}\left(\vartheta_{N, N+\left[n^{1 / 2}\right]} \theta_{N+j}\right)\right)=O(1)+O\left(C_{n}^{2} \sum_{j=0}^{\left[s^{2 / 3}\right]} \mathbf{E}\left(\vartheta_{N, N+\left[n^{1 / 2}\right]} \theta_{N+j}\right)\right)+ \\
+O\left(C_{n}^{2} \sum_{j=\left[s^{2 / 3}\right]+1}^{s-1} \mathbf{E}\left(\vartheta_{N, N+\left[n^{1 / 2}\right]} \theta_{N+j}\right)\right) .
\end{gathered}
$$

Заметим, что при $j=0, \ldots,\left[s^{2 / 3}\right]$ величины $\vartheta_{N, N+\left[n^{1 / 2}\right]} \theta_{N+j}$ равны нулю по определению. Кроме того, очевидно, что

$$
\vartheta_{N, N+\left[n^{1 / 2}\right]} \theta_{N+j} \leq \vartheta_{N, N+\left[n^{1 / 2}\right]} \mathbf{I}\left\{\mu\left(Y_{N+s}(j)\right)=j\right\},
$$

следовательно,

$$
C_{n}^{2} \sum_{j=\left[s^{2 / 3}\right]+1}^{s-1} \mathbf{E}\left(\vartheta_{N, N+\left[n^{1 / 2}\right]} \theta_{N+j}\right)=O\left(\sum_{j=\left[s^{2 / 3}\right]+1}^{s-1} \mathbf{P}\left\{\mu\left(Y_{N+s}(j)\right)=j\right\}\right)=o(1)
$$

Остается получить оценки для случая $\iota_{1} \in J^{(1)}, \iota_{2} \in J^{(1)}\left(\iota_{1}\right)$. Рассмотрим отдельно случаи $\iota_{2} \in J_{1}^{(1)}\left(\iota_{1}\right)$ и $\iota_{2} \in J_{2}^{(1)}\left(\iota_{1}\right)$. Пусть $\iota_{2} \in J_{1}^{(1)}\left(\iota_{1}\right)$. 
По аналогии с предыдущим случаем нетрудно убедиться в том, что

$$
\begin{gathered}
\sum_{\iota_{1} \in J^{(1)}} \sum_{\iota_{2} \in J_{1}^{(1)}\left(\iota_{1}\right)} \mathbf{E}\left(g_{\iota_{1}}^{\prime} g_{\iota_{2}}^{\prime}\right)=O\left(N^{1 / 3} C_{n}^{3}\left((N)_{s}\right)^{4} N^{-4 s}\right)+ \\
+O\left(C_{n}^{3} \sum_{j=0}^{s-1} \mathbf{E}\left(\vartheta_{N, N+\left[n^{1 / 2}\right]} \vartheta_{N+j, N+2\left[n^{1 / 2}\right]}\right)\right)
\end{gathered}
$$

причем

$$
O\left(N^{1 / 3} C_{n}^{3}\left((N)_{s}\right)^{4} N^{-4 s}\right)=o(1)
$$

Положим

$$
\vartheta_{i_{1}, i_{2}}(t)=\vartheta_{i_{1}, i_{2}} \mathbf{I}\left\{\mu\left(Y_{i_{2}}(s)\right)=s-t\right\}, 1 \leq t \leq t_{n},
$$

Ясно, что

$$
\vartheta_{i_{1}, i_{2}}=\sum_{t=1}^{t_{n}} \vartheta_{i_{1}, i_{2}}(t)
$$

При $j=0, \ldots, s-1$ справедливы оценки

$$
\begin{gathered}
\mathbf{E}\left(\vartheta_{N, N+\left[n^{1 / 2}\right]} \vartheta_{N+j, N+2\left[n^{1 / 2}\right]}\right)= \\
=\mathbf{E}\left(\left(\sum_{t=1}^{t_{n}} \vartheta_{N, N+\left[n^{1 / 2}\right]}(t)\right)\left(\sum_{l=1}^{t_{n}} \vartheta_{N+j, N+2\left[n^{1 / 2}\right]}(l)\right)\right) \leq \\
\leq \mathbf{E}\left(\vartheta_{N, N+\left[n^{1 / 2}\right]}(1) \vartheta_{N+j, N+2\left[n^{1 / 2}\right]}(1)\right)+ \\
+\mathbf{E}\left(2\left(\sum_{t=1}^{t_{n}} \vartheta_{N, N+\left[n^{1 / 2}\right]}(t)\right)\left(\sum_{l=2}^{t_{n}} \vartheta_{N+j, N+2\left[n^{1 / 2}\right]}(l)\right)\right) .
\end{gathered}
$$

Нетрудно показать (аналогичные рассуждения см. в доказательстве теоремы 1 статьи [8]), что

$$
\mathbf{E}\left(\left(\sum_{t=1}^{t_{n}} \vartheta_{N, N+\left[n^{1 / 2}\right]}(t)\right)\left(\sum_{l=2}^{t_{n}} \vartheta_{N+j, N+2\left[n^{1 / 2}\right]}(l)\right)\right) \leq \mathbf{E} \vartheta_{N, N+\left[n^{1 / 2}\right]} \kappa_{S},
$$

где

$$
\kappa_{s}=\max _{M \in M_{s}} \mathbf{P}\left\{\left(X_{1}, \ldots, X_{s}\right) \in M\right\} \leq N^{-s}(N)_{s-2},
$$

2012. T. 3. № 3. C. 129-151 
$M_{s}$ - множество классов структурной эквивалентности $s$-цепочек, содержащих не более $s-2$ различных символов. Поэтому при $n \rightarrow \infty$ справедливы оценки

$$
\begin{aligned}
& \mathbf{E}\left(\left(\sum_{t=1}^{t_{n}} \vartheta_{N, N+\left[n^{1 / 2}\right]}(t)\right)\left(\sum_{l=2}^{t_{n}} \vartheta_{N+j, N+2\left[n^{1 / 2}\right]}(l)\right)\right)= \\
= & O\left(N^{-2 s}\left((N)_{s}\right)^{2} N^{-s}(N)_{s-2}\right)=O\left(s^{-2} N^{-3 s}\left((N)_{s}\right)^{3}\right) .
\end{aligned}
$$

Оценим величины $\mathbf{E}\left(\vartheta_{N, N+\left[n^{1 / 2}\right]}(1) \vartheta_{N+j, N+2\left[n^{1 / 2}\right]}(1)\right)$. Из определения $\vartheta_{i_{1}, i_{2}}(1)$ следует, что цепочки $X_{N}, \ldots, X_{N+s-1}$ и $X_{N+j}, \ldots, X_{N+s+j-1}$ содержат одну пару совпадающих символов каждая. Рассмотрим два случая. Тогда

Пусть совпадающие символы принадлежат цепочке $X_{N+j}, \ldots, X_{N+s-1}$.

$$
\begin{gathered}
\mathbf{E}\left(\vartheta_{N, N+\left[n^{1 / 2}\right]}(1) \vartheta_{N+j, N+2\left[n^{1 / 2}\right]}(1) \mathbf{I}\left\{\mu\left(Y_{N+j}(s-j)\right)=s-j-1\right\}\right) \leq \\
\leq N^{-3 s-j} \sigma(s-j, s-j-1)\left((N)_{s-j-1}\right)^{3}\left((N-s+j+1)_{j}\right)^{4}= \\
=N^{-j}(N-s+j+1)_{j} N^{-3 s} C_{s-j}^{2}\left((N)_{s-1}\right)^{3} .
\end{gathered}
$$

Следовательно,

$$
\begin{gathered}
C_{n}^{3} \sum_{j=0}^{s-1} \mathbf{E}\left(\vartheta_{N, N+\left[n^{1 / 2}\right]}(1) \vartheta_{N+j, N+2\left[n^{1 / 2}\right]}(1) \mathbf{I}\left\{\mu\left(Y_{N+j}(s-j)\right)=s-j-1\right\}\right)= \\
=O\left(n^{3} s^{-1} N^{-3 s}\left((N)_{s}\right)^{3}\right) \sum_{j=0}^{s-1} N^{-j}(N-s+j+1)_{j}= \\
=O\left(s^{-1}\right)\left(\sum_{j=0}^{[s / 2]-1} N^{-j}(N-s+j+1)_{j}+\sum_{[s / 2]}^{s-1} N^{-j}(N-s+j+1)_{j}\right) .
\end{gathered}
$$

Заметим, что с ростом $j, j=0, \ldots, s-1$, величины $a_{j}=N^{-j}(N-$ $s+j+1)_{j}$ не возрастают, а именно,

$$
a_{j+1} a_{j}^{-1}=1-s N^{-1}+(j+2) N^{-1}, j=0, \ldots, s-2,
$$

причем при $j=0, \ldots,[s / 2]-1$ величина $a_{j+1} a_{j}^{-1}$ не превосходит $1-\frac{s}{2 N}$, поэтому

$$
\sum_{j=0}^{[s / 2]-1} N^{-j}(N-s+j+1)_{j} \leq \sum_{j=0}^{[s / 2]-1}\left(1-\frac{s}{2 N}\right)^{j}=O(1)
$$


и

$$
\sum_{j=[s / 2]}^{s-1} N^{-j}(N-s+j+1)_{j}<s\left(1-\frac{s}{2 N}\right)^{[s / 2]}=o(1)
$$

Пусть теперь совпадающие символы не принадлежат цепочке $X_{N+j}, \ldots, X_{N+s-1}$. Тогда мы имеем две различные пары совпадающих символов, которые принадлежат цепочкам $X_{N}, \ldots, X_{N+s-1}$ и $X_{N+j}, \ldots, X_{N+s+j-1}$ соответственно, причем для каждой пары по крайней мере один символ не принадлежит цепочке $X_{N+j}, \ldots, X_{N+s-1}$. Следовательно,

$$
\begin{gathered}
\mathbf{E}\left(\vartheta_{N, N+\left[n^{1 / 2}\right]}(1) \vartheta_{N+j, N+2\left[n^{1 / 2}\right]}(1) \mathbf{I}\left\{\mu\left(Y_{N+j}(s-j)\right)=s-j\right\}\right) \leq \\
\leq N^{-3 s-j}\left((N)_{s-j}\right)^{3}\left(\sigma(j, j-1)\left((N-s+j)_{j-1}\right)^{2}+j\left((N-s+j)_{j-1}\right)^{2}\right)^{2}= \\
=O\left(n^{3} N^{-3 s}\left((N)_{s}\right)^{3} s^{-1}\right) N^{-j}(N-s+j)_{j-1} .
\end{gathered}
$$

Отсюда по аналогии с первым случаем получаем оценку

$C_{n}^{3} \sum_{j=0}^{s-1} \mathbf{E}\left(\vartheta_{N, N+\left[n^{1 / 2}\right]}(1) \vartheta_{N+j, N+2\left[n^{1 / 2}\right]}(1) \mathbf{I}\left\{\mu\left(Y_{N+j}(s-j)\right)=s-j\right\}\right)=O\left(s^{-2}\right)$.

Таким образом, с учетом (18) получаем

$$
\sum_{\iota_{1} \in J^{(1)}} \sum_{\iota_{2} \in J_{1}^{(1)}\left(\iota_{1}\right)} \mathbf{E}\left(g_{\iota_{1}}^{\prime} g_{\iota_{2}}^{\prime}\right)=o(1)
$$

Рассмотрим последний случай: $\iota_{1}=\left(j_{1}, j_{2}, r_{1}\right) \in J^{(1)}, \iota_{2}=\left(i_{1}, i_{2}, r_{2}\right) \in$ $\in J_{2}^{(1)}\left(\iota_{1}\right)$. Пусть для определенности $i_{2}-j_{2}>i_{1}-j_{1} \geq 0$.

Рассмотрим сначала случай $i_{2}-j_{2} \leq\left[s^{3 / 4}\right]$. Пусть $X_{m}-$ крайний справа символ цепочки $Y_{j_{2}}(s)$, встретившийся в ней более одного раза. Из определения случайных величин $\vartheta_{j_{1}, j_{2}}$ следует, что $j_{2} \leq m<j_{2}+s-T_{n}<j_{2}+s-s^{[4 / 5]}$, а также что этот символ совпадает с некоторым символом $X_{l}, j_{2}+s^{[4 / 5]}<$ $<j_{2}+T_{n} \leq l<m$; значит, этот символ принадлежит цепочке $Y_{i_{2}}(s)$. Из структурной эквивалентности цепочек $Y_{j_{2}}(s)$ и $Y_{j_{1}}(s)$ и определения величины $\vartheta_{i_{1}, i_{2}}$ следует, что символ $X_{m+j_{1}-j_{2}}$ встретился в цепочках $Y_{j_{1}}(s)$ и $Y_{i_{1}}(s)$ также более одного раза, причем совпавшие с ними символы лежат на перекрытии этих цепочек. Тогда из структурной эквивалентности цепочек $Y_{i_{1}}(s)$ и $Y_{i_{2}}(s)$ следует, что символ $X_{m+j_{1}-j_{2}+i_{2}-i_{1}}$ встретится в цепочке $Y_{i_{2}}(s)$, а следовательно, и в цепочке $Y_{j_{2}}(s)$, более одного раза. Но неравенство $m+j_{1}-j_{2}+i_{2}-i_{1}>m$ 
противоречит определению числа $m$, значит, при $i_{2}-j_{2} \leq\left[s^{3 / 4}\right]$ справедливо равенство $\vartheta_{i_{1}, i_{2}} \vartheta_{j_{1}, j_{2}}=0$.

Пусть теперь $\left[s^{3 / 4}\right]<i_{2}-j_{2}$. Тогда из определения $\vartheta_{j_{1}, j_{2}}$ следует оценка

$$
\vartheta_{i_{1}, i_{2}} \vartheta_{j_{1}, j_{2}} \leq \vartheta_{j_{1}, j_{2}} \mathbf{I}\left\{\mu\left(Y_{j_{2}+s}\left(i_{2}-j_{2}\right)\right) \leq t_{n}\right\}
$$

Значит,

$$
\begin{gathered}
\mathbf{E} \vartheta_{i_{1}, i_{2}} \vartheta_{j_{1}, j_{2}}=O\left(N^{-2 s}\left((N)_{s}\right)^{2} \mathbf{P}\left\{\mu\left(Y_{j_{2}+s}\left(\left[s^{3 / 4}\right]\right)\right) \leq\left[s^{1 / 7}\right]\right\}\right)= \\
=O\left(N^{-2 s}\left((N)_{s}\right)^{2} e^{-s^{1 / 3}}\right) .
\end{gathered}
$$

Поэтому

$$
\sum_{\iota_{1} \in J^{(1)}} \sum_{\iota_{2} \in J_{2}^{(1)}\left(\iota_{1}\right)} \mathbf{E}\left(\gamma_{\iota_{1}} \gamma_{\iota_{2}}\right)=O\left(C_{n}^{2} s^{2} N^{-2 s}\left((N)_{s}\right)^{2} e^{-s^{1 / 3}}\right)=o(1) .
$$

Таким образом, доказано, что

$$
\rho\left(\mathcal{L}(\Gamma), \mathcal{L}\left(\Gamma^{\prime}\right)\right)=o(1)
$$

Следовательно, при $n \rightarrow \infty$ распределение случайного вектора $\left(\widehat{\xi}_{n}^{(12)}, \widehat{\eta}_{n}\right)$ сближается по вариации с распределением случайного вектора $\left(\xi_{n}^{\prime}, \eta_{n}^{\prime}\right)$ с независимыми компонентами, где

$$
\begin{gathered}
\xi_{n}^{\prime}=\sum_{q=1}^{\left[N^{1 / 3}\right]} q \sum_{\substack{N \leq i_{1}<i_{1}+\left[n^{1 / 2}\right] \leq i_{2} \leq n-N \\
\eta_{n}^{\prime}}} g_{i_{1}, i_{2}, q}^{(1)}, \\
\sum_{r=1}^{\left[N^{1 / 3}\right]} r \sum_{i=N+1}^{n-N} g_{i, r}^{(0)} .
\end{gathered}
$$

Докажем сходимость распределения случайной величины $\xi_{n}^{\prime}$ к распределению случайной величины $\xi$.

Заметим, что при $q \leq\left[N^{1 / 3}\right]$ событие $\vartheta_{i_{1}, i_{2}} \vartheta_{i_{1}+q-1, i_{2}+q-1}=1$ влечет за собой события $\vartheta_{i_{1}+r, i_{2}+r}=1, r=1, \ldots, q-2$ (это следует из определения 
$\left.\vartheta_{i_{1}, i_{2}}\right)$, поэтому

$$
\begin{gathered}
\sum_{N \leq i_{1}<i_{1}+\left[n^{1 / 2}\right] \leq i_{2} \leq n-N} \mathbf{E g}_{i_{1}, i_{2}, q}^{(1)}=C_{n}^{2}(1+o(1)) \times \\
\times \mathbf{P}\left\{\vartheta_{N, N+2 s}=0, \vartheta_{N+1, N+2 s+1}=\ldots=\vartheta_{N+r, N+2 s+r}=1,\right. \\
\left.\vartheta_{N+q+1, N+2 s+q+1}=0\right\}=C_{n}^{2}(1+o(1)) \mathbf{P}\left\{\vartheta_{N+1, N+2 s+1}=1\right\} \times \\
\mathbf{P}\left\{\vartheta_{N, N+2 s}=0, \vartheta_{N+q, N+2 s+q}=1, \vartheta_{N+q+1, N+2 s+q+1}=0 \mid \vartheta_{N+1, N+2 s+1}=1\right\} .
\end{gathered}
$$

Так как при $\vartheta_{N+1, N+2 s+1}=1$ цепочка $Y_{N+1}(s)$ содержит не менее $s-k_{n}=$ $s-\left[s^{1 / 7}\right]$ различных символов, то при $n \rightarrow \infty$

$$
\begin{gathered}
\mathbf{P}\left\{\vartheta_{N, N+2 s}=0, \vartheta_{N+1, N+2 s+1}=\ldots=\vartheta_{N+q, N+2 s+q}=1,\right. \\
\left.\vartheta_{N+q+1, N+2 s+q+1}=0\right\}= \\
=\left((N-s)_{q-1}\right)^{2} N^{-2(q-1)}\left(1-(N-s)^{2} N^{-2}\right)^{2}(1+o(1))= \\
=\left((1-\alpha)^{2}\right)^{-(q-1)}\left(1-(1-\alpha)^{2}\right)^{2}(1+o(1)) .
\end{gathered}
$$

Таким образом, с учетом оценки

$$
C_{n}^{2} \mathbf{P}\left\{\vartheta_{N+1, N+2 s+1}=1\right\}=\frac{1}{2} \lambda^{2}\left(e^{\frac{\alpha^{2}}{2(1-\alpha)^{2}}}-1\right)(1+o(1))
$$

получаем, что

$$
\begin{aligned}
& \sum_{N \leq i_{1}<i_{1}+\left[n^{1} / 2\right]<i_{2}<n-N} \mathbf{E} g_{i_{1}, i_{2}, q}^{(1)}=\frac{1}{2} \lambda^{2}\left(e^{\frac{\alpha^{2}}{2(1-\alpha)^{2}}}-1\right) \times \\
& \times\left((1-\alpha)^{2}\right)^{-(q-1)}\left(1-(1-\alpha)^{2}\right)^{2}(1+o(1)), \quad q=1,2 \ldots
\end{aligned}
$$

Сходимость распределения случайной величины $\eta_{n}^{\prime}$ к распределению случайной величины $\eta$ доказывается аналогично.

Таким образом, распределение случайного вектора $\left(\xi_{n}^{\prime}, \eta_{n}^{\prime}\right)$ сходится по вариации к распределению случайного вектора $(\xi, \eta)$, а значит, распределение случайной величины $\xi_{n}$ сходится к распределению суммы $\xi+C_{\eta}^{2}$.

Утверждения пп. а) теорем 2 и 3 доказаны. 
3. Докажем пп. б) этих теорем. Положим $k \geq 3$,

$$
\begin{gathered}
\xi_{n, k}^{(12)}=\sum_{\substack{1 \leq i_{1}<i_{1}+s \leq i_{2}<\ldots<i_{k-1}+s \leq i_{k} \leq n \\
\xi_{n, k}^{(13)}=}} \mathbf{I}\left\{S\left(Y_{i_{1}}(s)\right)=\ldots=S\left(Y_{i_{k}}(s)\right), \mu\left(Y_{i_{1}}(s)\right)<s\right\}, \\
\sum_{\substack{1 \leq i_{1}<i_{2}<\ldots<i_{k} \leq n, \exists j=1, \ldots, k-1: i_{j+1}-i_{j} \leq s-1}} \mathbf{I}\left\{S\left(Y_{i_{1}}(s)\right)=\ldots=S\left(Y_{i_{k}}(s)\right), \mu\left(Y_{i_{1}}(s)\right)<s\right\} .
\end{gathered}
$$

Очевидно, что

$$
\xi_{n, k}^{(1)}=\xi_{n, k}^{(12)}+\xi_{n, k}^{(13)} .
$$

Нетрудно видеть (см. также [7] ), что для случая $1 \leq i_{1}<i_{1}+s \leq$ $i_{2}<\ldots<i_{k-1}+s \leq i_{k} \leq n$ справедливо равенство

$$
\mathbf{P}\left\{S\left(Y_{i_{1}}(s)\right)=\ldots=S\left(Y_{i_{k}}(s)\right), \mu\left(Y_{i_{1}}(s)\right)<s\right\}=N^{-k s} \sum_{m=1}^{s-1} \sigma(s, m)\left((N)_{m}\right)^{k} .
$$

Следовательно,

$$
\begin{gathered}
\mathbf{E} \xi_{n, k}^{(12)} \leq C_{n}^{k} N^{-k s} \sum_{m=1}^{s-1} \sigma(s, m)\left((N)_{m}\right)^{k} \leq \\
\leq C_{n}^{k} N^{-k s}\left((N)_{s-1}\right)^{k-2} \sum_{m=1}^{s-1} \sigma(s, m)\left((N)_{m}\right)^{2} \leq \\
\leq(N-s+1)^{2-k}\left(n(N)_{s} N^{-s}\right)^{k-2} C_{n}^{2} N^{-2 s} \sum_{m=1}^{s-1} \sigma(s, m)\left((N)_{m}\right)^{2} .
\end{gathered}
$$

Выше (см. (12)) было доказано, что

$C_{n}^{2} N^{-2 s} \sum_{m=1}^{s-1} \sigma(s, m)\left((N)_{m}\right)^{2}=\frac{1}{2} \lambda^{2}\left(e^{\frac{\alpha^{2}}{2(1-\alpha)^{2}}}-1\right)(1+o(1))=O(1)$.

Следовательно,

$$
\mathbf{P}\left\{\xi_{n, k}^{(12)}>0\right\} \leq \mathbf{E} \xi_{n, k}^{(12)}=O\left((N-s+1)^{2-k}\right)=o(1) .
$$

Далее рассмотрим случайную величину $\xi_{n, k}^{(13)}$. Так как

$$
\left\{\xi_{n, k}^{(13)}>0\right\} \Rightarrow\left\{\xi_{n}^{(13)}>0\right\}, \quad k \geq 3
$$


то с учетом оценки (10) получаем

$$
\mathbf{P}\left\{\xi_{n, k}^{(13)}>0\right\} \leq \mathbf{P}\left\{\xi_{n}^{(13)}>0\right\} \leq \mathbf{E} \xi_{n}^{(13)}=o(1)
$$

Теоремы 2 и 3 доказаны.

\section{Список литературы}

1. Сачков В.Н. Введение в комбинаторные методы дискретной математики. - М. : Наука, 1982. - 384 с.

2. Буравлев С. М. Повторения с точностью до перестановок в последовательности независимых испытаний // Дискретная математика. - 1999. Т. 11. Вып. 1. - С. 53-75.

3. Ивченко Г.И., Медведев Ю.И. Асимптотические представления конечных разностей от степенной функции в произвольной точке // Теория вероятностей и ее применения. - 1965. - Т. 10. Вып. 1. - С. 151-156.

4. Михайлов В.Г. Об асимптотических свойствах распределения числа пар Н-связанных цепочек // Дискретная математика. - 2002. - Т. 14. Вып. 3. - C. 122-129.

5. Михайлов В.Г. Об асимптотическом поведении вероятности наличия в последовательности эквивалентных цепочек с нетривиальной структурой // Дискретная математика. - 2008. - Т. 20. Вып. 4. С. $113-119$.

6. Михайлов В.Г., Шойтов А. М. Структурная эквивалентность $s$-цепочек в случайных дискретных последовательностях // Дискретная математика. - 2003. - Т. 15. Вып. 4. - С. 7-34.

7. Шойтов А.М. Предельные распределения числа наборов Н-эквивалентных отрезков в равновероятной полиномиальной схеме серий // Дискретная математика. - 2002. - Т. 14. Вып. 1. - С. 82-98.

8. Шойтов A. М. Пуассоновское приближение для числа повторений значений дискретной функции от цепочек // Дискретная математика. -2005. - Т. 17. Вып. 2. - С. 56-69.

9. Шойтов А.М. Дискретные предельные распределения для числа цепочек с одинаковой структурой в последовательности равновероятных испытаний // Обозрение прикладной и промышленной математики. 2007. - Т. 14. Вып. 3. - C. 474-484.

10. Barbour A. D., Holst L., Janson S. Poisson Approximation. - Oxford: Oxford Univ. Press, 1992. - 277 p. 